Se nourrir des souffles et des saveurs : la diététique de la médecine chinoise

Éric MARIE

\author{
(2) OpenEdition \\ Journals \\ Édition électronique \\ URL : http://journals.openedition.org/transtexts/602 \\ DOI : $10.4000 /$ transtexts.602 \\ ISSN : 2105-2549 \\ Éditeur \\ Gregory B. Lee
}

Référence électronique

Éric MARIE, "Se nourrir des souffles et des saveurs : la diététique de la médecine chinoise », Transtext (e)s Transcultures 跨文本跨文化 [En ligne], 10 | 2015, mis en ligne le 09 novembre 2016, consulté le 19 avril 2019. URL : http://journals.openedition.org/transtexts/602 ; DOI : 10.4000/transtexts.602

Ce document a été généré automatiquement le 19 avril 2019

(c) Tous droits réservés 


\title{
Se nourrir des souffles et des saveurs : la diététique de la médecine chinoise
}

\author{
Éric MARIE
}

1 La médecine traditionnelle chinoise présente une double particularité : elle a conservé, sur une très longue durée, une remarquable continuité épistémologique et elle bénéficie d'un statut institutionnel de médecine officielle dans son pays d'origine. Bien plus qu'un ensemble de techniques, il s'agit d'un vaste système incluant de nombreuses branches thérapeutiques. Si les plus connues, telles que la pharmacopée et l'acupuncture, sont largement pratiquées en milieu hospitalier, elle intègre également des pratiques pouvant trouver leur place dans le cadre familial ou populaire qui visent aussi bien à traiter les maladies qu'à entretenir la santé et à améliorer les fonctions vitales.

2 La diététique participe à cette finalité et joue un rôle essentiel dans la conception chinoise de l'équilibre de l'individu dans ses rapports avec l'environnement. Le terme "diététique " peut cependant prêter à confusion car les théories et les pratiques dont il est question sont très différentes de celles qui relèvent de cette discipline en Occident. Loin d'être fondé sur un ensemble de recommandations et d'interdictions, comme on en rencontre dans tant d'écoles qui divisent les ingrédients en leur attribuant des qualités ou des défauts intrinsèques, le système chinois intègre pratiquement toutes les catégories d'aliments et se fonde sur une individualisation de la prescription, ce qui est conseillé à une personne pouvant s'avérer nuisible à une autre. Les règles qui prévalent sont notamment fondées sur la nature complexe des énergies et saveurs, tropismes et actions spécifiques des ingrédients et de leurs combinaisons, ainsi que sur leurs relations avec la manière dont on perçoit la physiologie humaine dans la pensée médicale chinoise.

\section{Sources et histoires de la diététique chinoise}

3 Il est difficile de situer précisément l'origine des pratiques diététiques en Chine. On fait souvent remonter à l'empereur légendaire Shennong la découverte des bases de 
l'agriculture, l'usage de la pharmacopée et de la diétothérapie. Cependant, le nom de ce fondateur mythique n'apparait pour la première fois que dans le Huainanzi 淮南子 [Les maitres de Huainan], un texte du IIe siècle avant J.-C. Dans le Tianguan zhonzai 天官冢宰 [Offices en rapport avec le ciel, premier ministère] du Zhouli 周禮 [Rites des Zhou], ouvrage qui répertorie les fonctions officielles sous la dynastie des Zhou (1122-256 avant J.-C., selon la chronologie traditionnelle), la médecine comporte quatre spécialités bien identifiées ${ }^{1}$ : jiyi 疾醫 [médecine générale], shiyi 食醫 [diététique] yangyi 瘍醫 [chirurgie] ${ }^{2}$, qui inclut également le traitement des maladies de la peau ainsi que l'orthopédie, et shouyi 獸醫 [médecine vétérinaire]. Les diététiciens de cette époque sont souvent présentés comme les premiers praticiens d'un art de la santé fondé sur la prévention et le traitement par l'équilibre alimentaire. Leur fonction consistait notamment à préserver la santé du souverain par l'application des principes de yangsheng 養生 [entretien du principe vital].

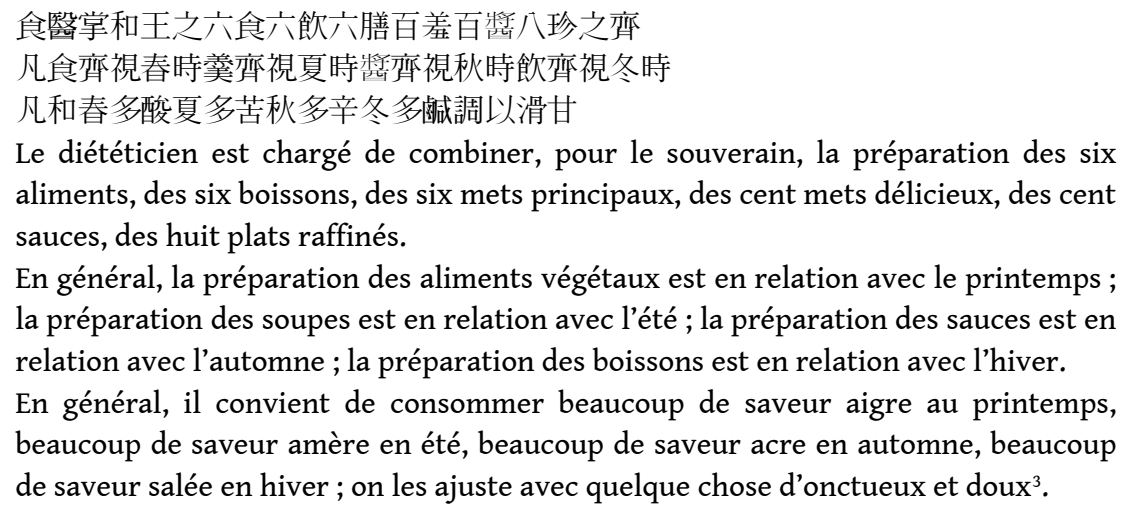

4 Ce passage montre le rôle du diététicien qui indique à l'intendant des repas du souverain comment il convient d'associer les saveurs et comment les mets doivent être " assaisonnés ", au sens originel du terme, c'est-à-dire adaptés à la saison, ici, selon la règle des wuxing 五行 [cinq mouvements].

Les aspects les plus anciens du système savant de la médecine traditionnelle chinoise, tel qu'il apparaît à travers sa littérature classique, se constituent surtout à partir du IIIe siècle avant J.-C. En dehors des légendes et de quelques citations éparses provenant de sources non médicales, nous ne possédons que peu d'indices sur les pratiques médicales antérieures à cette date. Le principal texte fondateur de la médecine chinoise est le Huangdi neijing 黃帝内徑 [Classique interne de l'Empereur jaune], ouvrage composite dont la rédaction s'est étalée sur plusieurs siècles, avec un grand nombre de pertes, ajouts et remaniements, pour atteindre, à partir du VIIe siècle de notre ère, la forme que nous en connaissons aujourd'hui. S'il ne contient que quelques prescriptions contenant notamment des ingrédients alimentaires, il expose les règles de base communes à la diététique et à la pharmacopée, notamment les théories sur les saveurs. Il est à noter qu'il n'existe pas réellement de frontière entre aliments et médicaments dans le système médical chinois. Tout au long de l'histoire, les matières médicales incluent aussi bien des substances d'usage strictement thérapeutique et des ingrédients entrant couramment dans la cuisine familiale, tels que l'ail, le gingembre, la ciboule... La plus ancienne matière médicale qui nous soit parvenue, probablement rédigée autour du premier siècle avant J.C., le Shennong bencaojing 神農本草經 [Herbier classique de Shennong] comporte 365 substances, avec la mention de leur lieu d'origine, de leur préparation, de leurs saveurs et nature et de leurs actions thérapeutiques. Un grand nombre de ces ingrédients, principalement d'origine végétale, sont d'un usage alimentaire courant. En outre, il est 
fréquent d'intégrer des aliments dans des formules médicales. Un des plus anciens traités de thérapeutique, le Shanghan zabing lun 傷寒雜病論 [Traité des attaques du froid et de diverses maladies], rédigé par le fameux médecin Zhang Zhongjing 張仲景 vers l'an 200 de notre ère, et remanié ultérieurement sous la forme de deux livres distincts ${ }^{4}$, comprend des dizaines d'exemples d'associations de ce genre où le jaune d'œuf, le gingembre, le riz, le vinaigre, le miel et diverses viandes sont mélangés à des drogues d'action puissante. Cette tradition s'est perpétuée jusqu'à aujourd'hui et il est possible de commander, dans de nombreux restaurants de Chine, des plats traditionnels contenant des remèdes de la pharmacopée.

6 Sous les Tang (618-907), la diététique se développe et occupe une place plus importante dans la littérature médicale. Sun Simiao 孙思邀, (581-682), un des plus célèbres médecins de l'histoire de la Chine, rédige deux traités, le Beiji qianjin yaofang 備急千金要方 [Prescriptions essentielles d'urgence valant mille onces d'or] et le Qianjin yifang 千金翼 方 [Supplément aux prescriptions valant mille onces d'or] qui comprennent un grand nombre de considérations et de traitements diététiques. C'est à cette époque, entre 701 et 704, que Meng Shen 孟诜 écrit le premier recueil entièrement consacré à l'aspect médical de l'alimentation, le Shiliao bencao 食疗本草 [Matière médicale de diététique ${ }^{5}$, ouvrage de référence pour les générations ultérieures.

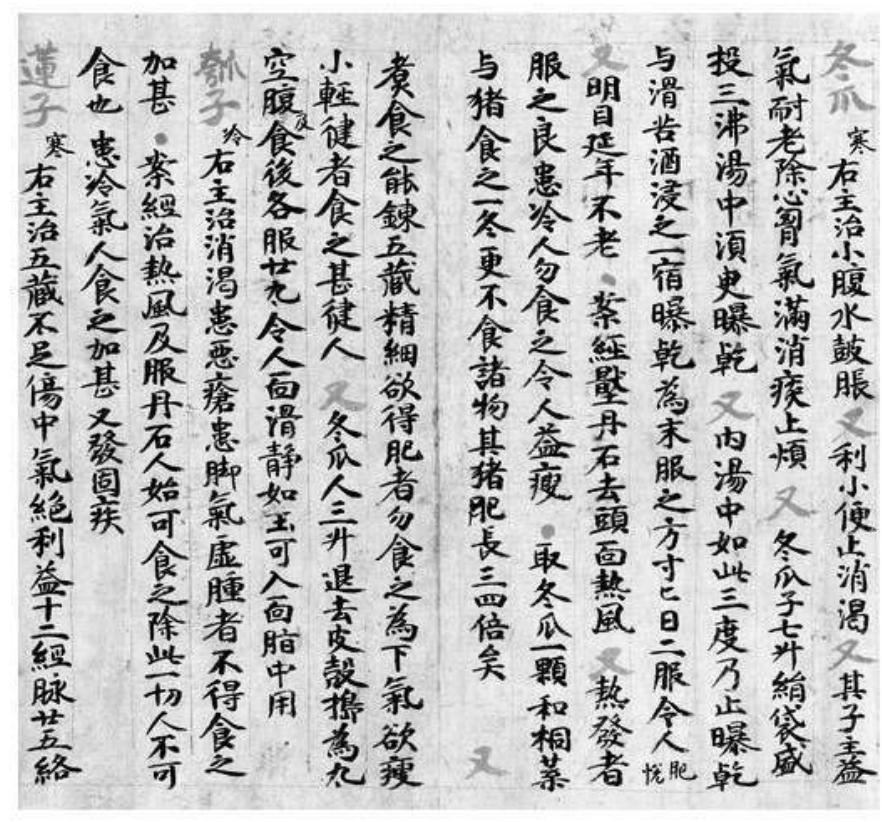

Fragment du Shiliao bencao 食疗本草 de Meng Shen, manuscrit découvert dans la grotte de Dunhuang.

7 Durant les dynasties suivantes des Song du Nord aux Song du Sud (960-1279), de vastes recueils de formules de pharmacopée sont rédigés. La diététique est de mieux en mieux prise en considération par les patients et les médecins. En gérontologie, par exemple, Chen Zhi 陳直, dans son livre Yanglaoshou xinshu 養老壽新書 [Nouvel ouvrage pour bien s'occuper de ses parents et leur assurer une longue vie] consacre une part importante à l'alimentation. La plus grande partie des recettes (environ $70 \%$ ) est composée de 
prescriptions diététiques. Au XIIIe siècle, les Mongols envahissent la Chine et prennent le pouvoir en fondant la dynastie Yuan (1279-1368). C'est à cette période que vécut Hu Sihui 忽思慧. D'origine mongole, il est médecin de l'empereur et rédige un des plus importants traités sur la question de l'alimentation: le Yinshan zhengyao 飲善正要 [Principes de diététique], publié en 1330. Ce livre décrit 230 ingrédients (céréales, légumes, fruits, viandes, poissons...), de nombreuses recettes, certaines incluant des remèdes de pharmacopée; il évoque les aliments déconseillés à certaines catégories de personnes ainsi que ceux qu'il faut éviter pendant la grossesse, l'allaitement... En outre, il insiste sur la tempérance et sur diverses règles d'hygiène.

8 Le plus célèbre médecin des Ming (1368-1644) est incontestablement Li Shizhen 李時珍 (1518-1593) dont l'œuvre la plus connue est le Bencao gangmu 本草綱目 [Compendium général de la matière médicale]. Il s'agit d'une somme magistrale contenant la présentation très détaillée de 1892 substances et comprenant de nombreuses références à la diététique, notamment plusieurs dizaines de traitements à base de céréales et autant de recettes de vins médicinaux. Sous les Qing (1644-1911), beaucoup de traités de diététique sont publiés. Le nombre d'ingrédients alimentaires décrits selon des critères médicaux dépasse les 300, avec Wang Shixiong 王士雄 (1808-1866). Les recettes se multiplient. La tendance à la compilation et à l'encyclopédisme pousse à préserver et reproduire les écrits antérieurs.

9 À l'époque contemporaine, des traités anciens sont redécouverts et les contributions à la diététique sont trop nombreuses pour être citées. Elles comprennent des matières médicales d'ingrédients ${ }^{6}$, des recueils de recettes populaires, des traités savants, des études spécialisées sur des aspects spécifiques tels que les thés ou les vins médicinaux. Plusieurs hôpitaux ont créé des départements de diététique, des unités de recherche procèdent à des investigations sur les substances traditionnelles de l'alimentation et on peut observer l'ouverture de restaurants diététiques ${ }^{7}$ où des dizaines de plats, répertoriés selon leurs propriétés thérapeutiques, sont proposés aux clients soucieux de contribuer ainsi à l'entretien de leur santé ou au traitement de leurs pathologies. 


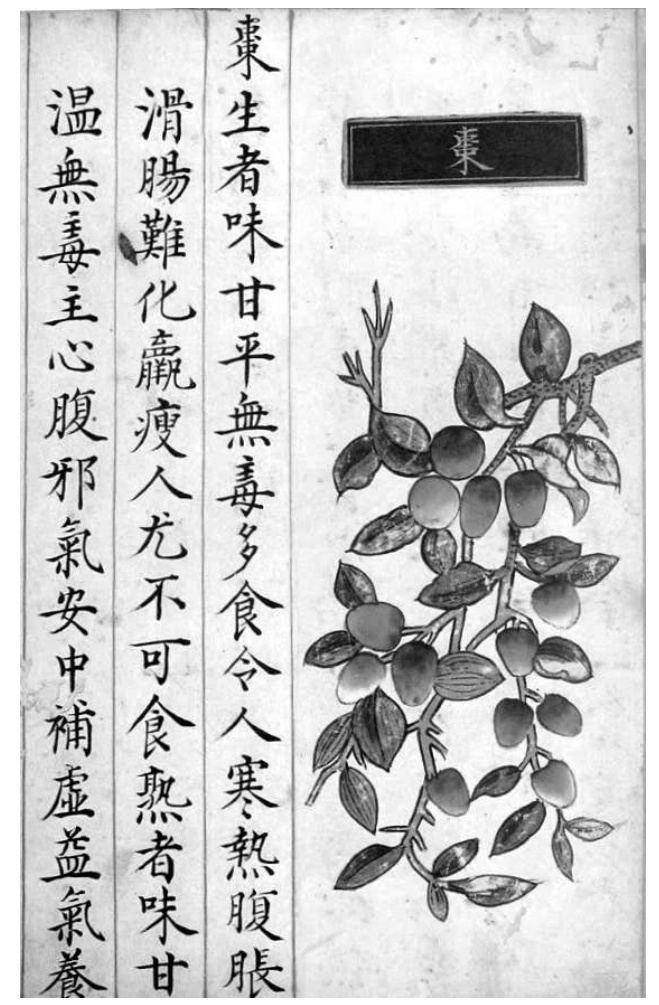

Jujube. Planche du Shiwu bencao食物本草 ${ }^{8}$,

\section{Principes fondamentaux de la diététique chinoise}

La diététique chinoise contemporaine s'appuie sur plusieurs grands principes. Le premier est qu'il n'existe pas de régime alimentaire idéal pouvant être appliqué à tout le monde. La prescription de conseils nutritionnels passe donc préalablement par un diagnostic différentiel, aussi précis que possible, et s'appuyant sur les règles et la nosologie particulières de la médecine traditionnelle chinoise. On ne peut donc pas élaborer un traitement diététique de l'hypertension, de l'obésité ou du diabète, en se fondant seulement sur les classifications de la médecine occidentale. La connaissance préalable des théories fondamentales et du diagnostic spécifiques de la médecine chinoise est indispensable. En effet, pour chaque maladie (bing 病), il existe un certain nombre de syndromes (zheng 證) en fonction desquels s'élabore la stratégie thérapeutique. Même des patients en bonne santé et souhaitant seulement quelques conseils préventifs peuvent relever de constitutions différentes qu'il faut savoir discerner. D'autre part, il faut considérer que la diététique peut être une méthode très efficace pour entretenir la santé et traiter de nombreux déséquilibres mais que son action s'opère généralement de façon progressive plutôt que dans l'urgence. En conséquence, le régime doit se fonder sur une alimentation agréable et réaliste sur le plan pratique. Si l'adage liangyao kukou 良藥苦口 [excellent traitement, saveur amère], qui signifie qu'un médicament efficace a souvent un goût désagréable, est accepté par les patients pendant une période courte et pour une petite quantité de substances médicinales rapidement absorbée, il est difficile de se nourrir quotidiennement d'aliments de saveur répulsive. Au contraire, la diététique chinoise se fonde sur une réconciliation entre gastronomie et santé. Il s'agit d'encourager le patient à retrouver le lien avec les perceptions visuelles, olfactives et gustatives - 
plusieurs sens intervenant dans le plaisir de manger - qui lui sont favorables. Pour agir ainsi, le diététicien doit posséder une bonne connaissance des ingrédients et de leurs combinaisons. Ce savoir ne repose pas seulement, comme en Occident, sur la composition chimique des aliments - bien que celle-ci soit mentionnée dans la plupart des ouvrages chinois contemporains - mais il s'appuie sur une connaissance de caractéristiques très différentes. Il suffit, pour le constater, d'observer comment sont présentées les matières médicales des aliments. Les substances alimentaires sont classées en catégories globales, comme on pourrait les trouver réparties dans un magasin ou sur un marché (fruits, légumes, céréales, viandes, poissons, vins, thés, condiments...) et chaque ingrédient est présenté sous forme d'une fiche regroupant les informations essentielles qui définissent son usage.

Caractéristiques des aliments selon la diététique chinoise

Dans la plupart des matières médicales de diététique, on trouve les rubriques suivantes :

- Identification: dénomination (avec souvent la mention de plusieurs noms) et description sommaire.

- Lieu et période de production, le cas échéant.

15 - Nature (xing 性): exprime l'action de réchauffement ou de refroidissement qui se décline en cinq degrés (chaud, tiède, neutre, frais, froid).

16 - Saveur (wei 味) : on dénombre cinq saveurs principales (aigre ou acide, amer, doux, acre ou piquant, salé) et deux saveurs secondaires (insipide ou fade, astringent).

17 - Tropisme (guijing 歸經) : désigne le lieu d'action dans le corps.

18 - Action physiologique induite.

19 - Indications thérapeutiques : liste de maladies et symptômes que l'aliment peut soigner, généralement dans une recette composée avec d'autres ingrédients qui interagissent de multiples manières.

20 - Contre-indications éventuelles.

21 - Modes de préparation recommandés: désigne notamment les actions mécaniques (broyer, découper...) et l'utilisation combinée de l'eau et du feu dans la cuisson (griller à sec, frire, ébouillanter...).

22 - Exemples de recettes.

23 On peut également mentionner, bien qu'elle ne fasse pas partie de la liste des caractéristiques traditionnelles, la forme (xing 形) qui ne fait pas seulement référence à la morphologie de l'ingrédient mais également à sa couleur et sa consistance (mou, croquant, fibreux...), les perceptions visuelles et tactiles étant supposées exercer une influence sur celui qui le consomme.

Nature des aliments et mouvements du Qi

La nature (xing 性) est fondée sur les effets produits sur le corps en termes d'opposition entre froid et chaleur, qui sont deux expressions fondamentales issues de la théorie du yinyang. Les aliments sont classés en cinq grandes catégories, selon cette dialectique : re 熱 [chaud], wen 溫 [tiède], ping 平 [neutre], liang 凉 [frais] et han 寒 [froid]. Ces termes ne désignent pas ici la température physique mais le dynamisme exercé par la substance sur le corps. Ainsi, un aliment de nature froide soutient le Yin et combat les effets d'une chaleur pathogène perceptible grâce à une investigation clinique fondée sur les règles du diagnostic chinois. Inversement, une substance de nature chaude combat le froid corporel 
et tonifie le Yang. Le frais et le tiède sont des expressions modérées du froid et du chaud. Cependant, au-delà de leur caractère quantitativement plus modéré, leur mode d'action est souvent analysé comme qualitativement différent. Ainsi, ce qu'on qualifie de chaud a souvent une action plus concentrée, comme un chalumeau, tandis que le tiède est plus diffus, agissant à la manière d'un radiateur. Ces critères relèvent directement de la dialectique du Qi 氣, concept chinois difficile à traduire du fait de son champ sémantique étendu et de l'absence d'équivalent dans la langue française ${ }^{9}$. Les natures des aliments ne constituent pas le seul critère en relation avec les fonctions du Qi. Celui-ci, par l'intermédiaire de l'activité physiologique des zangfu 藏腑 [organes et entrailles], exprime ses mouvements selon quatre directions : montée, descente, extériorisation et intériorisation. Lorsque l'activité viscérale est harmonieuse, aucune prédominance n'apparaît et tous les substrats corporels circulent de façon fluide, chacun suivant la voie que la nature lui désigne. Mais dans un processus morbide, ce dynamisme peut se dérégler. Ainsi, une altération des mouvements de montée et de descente peut produire prolapsus ou diarrhées (insuffisance de montée), dyspnée, accumulation de mucosités ou constipation (insuffisance de descente), céphalées ou vertiges (excès de montée) ou encore dysenterie (excès de descente). Les mécanismes d'intériorisation et d'extériorisation peuvent également être perturbés: lorsque l'intériorisation est excessive, les agents pathogènes externes pénètrent facilement et se bloquent à l'intérieur du corps, tandis que dans le cas contraire d'extériorisation excessive, le Qi correct du corps s'échappe facilement avec la transpiration qui est profuse. Pour réguler ce mécanisme, le médecin chinois utilise des substances qui s'opposent ou compensent ces mouvements perturbés. Certains ingrédients sont donc caractérisés par un mouvement directionnel qu'ils peuvent exercer sur le corps, selon que leur dynamisme est sheng 昇 [ascendant] (s'oppose à la descente), jiang 降 [descendant] (s'oppose à la montée), fu 浮 [émergent] (s'oppose à la pénétration de l'agent pathogène) ou chen 沉 [immergent] (retient l'échappement du Qi correct) ${ }^{10}$.

\section{Gouvernement du corps et alchimie des saveurs}

Pour comprendre les conceptions de la diététique chinoise, il faut savoir que dans la plupart des aspects de la médecine savante qui s'est élaborée en Chine, le corps humain est perçu comme l'Empire. Les viscères (zangfu 臟腑, littéralement «organes et entrailles ») ne sont pas des groupes de tissus mais des ministères et des administrations au service d'un gouvernement. On emploie d'ailleurs volontiers les termes d'empereur, de chancelier, de général, d'intendant ou de divers fonctionnaires pour les définir. Ils n'exercent pas seulement une action physiologique, ils "gouvernent ». On dira, par exemple : gan zhu shuxie 肝主疏舄 [le foie gouverne le drainage et la dispersion]. Il ne s'agit pas tant d'une fonction organique que d'une charge politique. De la même façon, on parlera de correspondances, de communication, de domination, d'attaque, toutes expressions utilisées pour définir des actions d'organisation sociale ou de stratégie militaire. Il est intéressant de noter que le sinogramme zhi 治 peut aussi bien signifier " gérer, administrer » que " soigner ». Il faut rappeler que, dans la Chine ancienne, la profession médicale n'a pas toujours constitué un métier à part entière, exercé pendant toute la vie. De nombreux médecins historiques ont occupé, en tant que lettrés, diverses fonctions administratives, politiques ou militaires. Ce facteur, associé à la conception du corps comme une représentation miniaturisée d'un empire, est sans doute à l'origine de 
cette terminologie. Or, dans la physiologie chinoise, les saveurs (wei 味) ne sont pas seulement considérées comme des perceptions liées au sens du goût, elles ont, au contraire, une action sur l'ensemble de l'organisme. Ainsi, lorsqu'on constate que la saveur âcre ou piquante, qu'on trouve, par exemple, dans le poivre ou le piment, provoque la sudorification, ce n'est pas seulement parce que le sujet ressent cette sensation au niveau buccal qu'il transpire. D'ailleurs, lorsqu'une formule à dominante piquante est administrée sous une forme qui échappe à la perception gustative - dans une gélule fermée, directement avalée, par exemple - l'effet sudorifique est obtenu, bien que le sujet ne perçoive pas la saveur. Les Chinois en ont déduit que les saveurs des aliments ingérés se définissaient davantage en fonction de l'effet physiologique observé que selon la sensation gustative ressentie subjectivement. Il en découle qu'une substance alimentaire ou pharmacologique est qualifiée de piquante, non seulement lorsqu'elle est perçue comme telle mais également lorsqu'elle produit les effets du piquant, quand bien même elle ne serait pas identifiée ainsi par le goût. Les conséquences de cette conception sont importantes car elle conduit à considérer que les saveurs sont perçues par l'ensemble du corps et qu'elles s'exercent par le biais des organes (zang 藏).

Ces saveurs sont au nombre de sept: xin 辛 [acre ou piquant], gan 甘 [doux], suan 酸 [acide ou aigre], ku 苦 [amer], xian 鰔 [salé], se 涩䜧 [astringent] et dan 淡 [insipide, fade]. Les cinq premières sont considérées comme prépondérantes car elles sont en relation avec les cinq organes principaux, selon la classification des wuxing 五行 [cinq mouvements]. Leur fonction globale est définie dans le Huangdi neijing, dans une vision ternaire de l'univers (ciel, terre, homme), en parallèle avec les climats et les émotions.

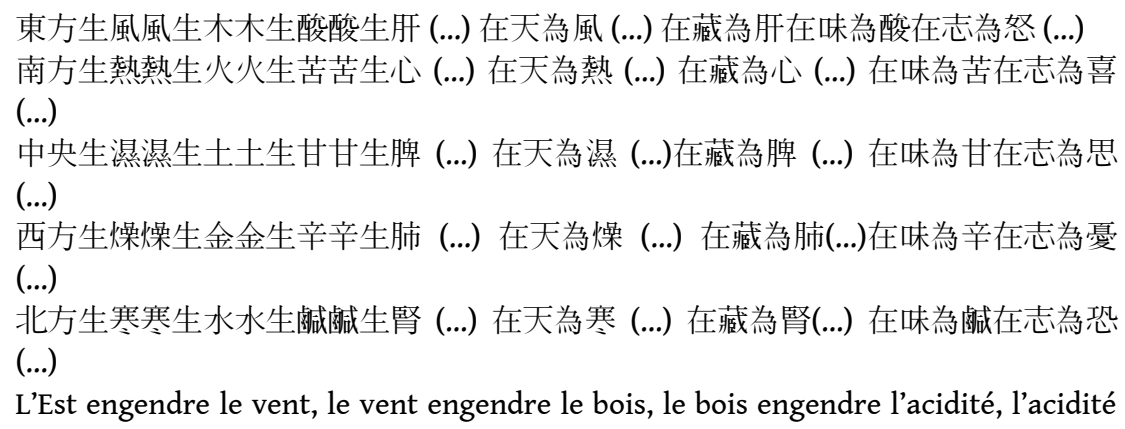
l'amertume engendre le cœur (...). Dans le ciel, c'est la chaleur (...) dans les organes, c'est le cœur ; dans les saveurs, c'est l'amertume ; dans les émotions, c'est la joie (...) Le Centre engendre l'humidité, l'humidité engendre la terre, la terre engendre le doux, le doux engendre la rate ${ }^{11}$ (...). Dans le ciel, c'est l'humidité (...) dans les organes, c'est la rate (...) dans les saveurs, c'est le doux ; dans les émotions, c'est la pensée (...)

L'Ouest engendre la sécheresse, la sécheresse engendre le métal, le métal engendre l'âcreté, l'âcreté engendre le poumon (...). Dans le ciel, c'est la sécheresse (...) dans les organes, c'est le poumon ${ }^{12}(. .$.$) dans les saveurs, c'est l'âcreté ; dans les émotions,$ c'est la tristesse (...)

Le Nord engendre le froid, le froid engendre l'eau, l'eau engendre le salé, le salé engendre le poumon (...). Dans le ciel, c'est l'eau (...) dans les organes, ce sont les reins (...) dans les saveurs, c'est le salé ; dans les émotions, c'est la peur (...) ${ }^{13}$

On peut résumer ce système de correspondances dans un tableau : 


\begin{tabular}{|l|l|l|l|l|l|}
\hline & Bois & Feu & Terre & Métal & Eau \\
\hline Ciel (climat) & Vent & Chaleur & Humidité & Sécheresse & Froid \\
\hline Terre (saveur) & Acide & Amer & Doux & Acre & Salé \\
\hline Homme (émotion) & Colère & Joie & Introspection & Tristesse & Peur \\
\hline Organe & Foie & Cœur & Rate & Poumon & Reins \\
\hline
\end{tabular}

29 Les relations entre les saveurs et les organes relèvent de modalités multiples. Tout d'abord, chaque saveur entre (ru 入) dans l'organe qui lui correspond. Ainsi, l'acidité entre dans le foie. Elle exerce ensuite une action ciblée sur la forme (xing 形) de l'organe concerné qu'elle nourrit ${ }^{14}$ lorsqu'elle est absorbée en quantité modérée mais qu'elle blesse ${ }^{15}$ lorsqu'elle est consommée en excès. Si cet excès est chronique, elle finit par nuire à d'autres organes. D'autre part, l'action de chaque saveur s'exerce sur le Qi des organes, selon des règles précises : elle disperse (xie 潟) le Qi de l'organe qui lui correspond et tonifie (bu 補) celui de l'organe opposé. Enfin, elle harmonise un autre organe lorsque celui-ci exprime une souffrance spécifique liée à un excès de son activité spécifique. Il faudrait un long développement pour exposer de façon exhaustive les interactions entre l'ensemble des saveurs et des organes mais on peut l'illustrer à partir d'une saveur, par exemple l'acide : cette saveur nourrit la forme du foie en quantité modérée, elle lui nuit en quantité excessive, de plus, elle peut également blesser la forme de la rate; elle disperse le $\mathrm{Qi} \mathrm{du}$ foie tandis qu'elle tonifie celui du poumon; enfin elle permet d'harmoniser le Qi du cœur lorsque celui-ci souffre d'un excès de relâchement en favorisant sa contraction.

30 Chaque saveur possède, en outre, un ou plusieurs effets spécifiques qui s'expriment sur l'ensemble des fonctions et substrats de l'organisme :

31 - L'acide peut collecter et restreindre (neng shou, neng se 能收、能歰). Il lutte contre l'échappement des fluides par son pouvoir astringent qui s'exerce à travers des effets physiologiques divers : antisudoral, antidiurétique, antileucorrhéique...

32 - L'amer peut purger (ou drainer), assécher et affermir (neng xie, neng zao, neng jian 能 泄、能燥、能堅). Il permet de purifier en favorisant le mouvement de descente du poumon et de l'estomac, d'éliminer l'Humidité pathogène dans de nombreux syndromes et de consolider le Yin des reins, notamment.

33 - Le doux peut tonifier, relâcher et harmoniser (neng bu, neng huan, neng he 能補、能 緩、能和). Il est nutritif, antispasmodique et il joue souvent un rôle de médiateur pour équilibrer les autres ingrédients d'une recette.

34 - L'âcre (piquant) peut disperser et mobiliser (能散、能行). Il dissipe les agents pathogènes d'origine externe par la sudorification, fait circuler le Qi, le sang et les fluides corporels; s'il s'agit d'un ingrédient aromatique, il transforme l'Humidité trouble (souvent générée par une mauvaise digestion).

35 - Le salé peut ramollir et purger par une action laxative qui favorise l'évacuation intestinale (neng ruan, neng xia 能軟、能下). Il assouplit les indurations et favorise le transit par humidification. 

et substrats corporels, prévenant leur échappement, et l'insipide (fade) exerce une action diurétique.

\section{Tropisme et méridien destinataire} couramment employé est guijing 歸經, qu'on traduit souvent par «méridien destinataire ${ }^{16}$ mais qui a un sens plus étendu. En effet, l'aliment agit non seulement sur les méridiens concernés, mais également sur les viscères correspondants, ceux-ci communiquant naturellement avec ceux-là. L'expression guijing apparaît sous les Qing (1644-1911), dans un traité de Shen Jin'ao 沈金鰲 publié en 1713, le Yaoyao fenji 要藥分劑 [Distinguer et prescrire les principales drogues], ouvrage qui présente et décrit 420 substances médicinales en les classant en dix catégories. Cependant, bien que le terme guijing soit d'usage relativement tardif, le principe consistant à caractériser les ingrédients, tant médicinaux qu'alimentaires, est beaucoup plus ancien et apparaît dans les traités classiques sous diverses expressions désignant le lieu de pénétration physiologique. C'est souvent l'action thérapeutique qui permet de comprendre ce tropisme. Ainsi, la noix qui «se rend aux reins et aux poumons " permet de traiter les tableaux cliniques d'asthme et de dyspnée attribués au manque de communication entre ces deux organes, avec une combinaison de symptômes respiratoires et de lombalgie et troubles urinaires.

Il faudrait encore évoquer la préparation mécanique (découpage, broyage...) des aliments et leurs nombreux modes de cuisson qui exercent une influence déterminante sur la nature finale. Enfin, les mélanges et combinaisons des ingrédients font l'objet de règles qui induisent l'action thérapeutique finale du plat qui sera consommé.

Ainsi, lorsqu'on étudie la diététique chinoise, elle se révèle sous la forme d'une discipline très élaborée dont la complexité conduit à une véritable alchimie des souffles et des saveurs, permettant à l'être humain d'établir, par une implication de la totalité de son corps, une relation sensorielle subtile avec son alimentation.

\section{NOTES}

1. . Il est difficile de dater précisément l'origine de cette division en spécialités car il est probable que le Zhouli n'ait pas été rédigé sous les Zhou mais au début des Han et qu'il ait été conçu comme une sorte de représentation rétrospective idéalisée de l'organisation sociale des Zhou.

2. . En fait, le terme peut se traduire littéralement par « médecine des ulcères ».

3. . Zhouli 周禮 [Rites des Zhou], Tianguan zhongzai 天官冢宰 [Offices en rapport avec le ciel, premier ministère], 5 .

4. . L'ouvrage est perdu, sous sa forme initiale, dès la fin des Han. Il réapparait sous la forme des deux principaux traités qui en sont issus et qui ont été reconstitués ou compilés ultérieurement pour atteindre, sous les Song, la forme que nous en connaissons aujourd'hui : le Shanghanlun 傷

Transtext(e)s Transcultures 跨文本跨文化, 10 | 2015 
寒論 [Traité des attaques du Froid] et le Jingui yaolüe fanglun 金貴要略方論 [Formulaire des prescriptions essentielles du coffre d'or].

5. . L'ouvrage ayant disparu, son contenu était incertain jusqu'à ce que Ma Jixing et à Xie Haizhou le reconstituent, permettant sa publication en 1984.

6. . Ye Juquan 叶粫泉 publie notamment un ouvrage de référence en 1978 : Shiwu zhongyao yu bianfang 食物中藥與便方 [Matière médicale et formulaire pratique des aliments], Shanghai, Zhongguo zhongyiyao chubanshe, 1984. Il contient la description de183 sortes d'ingrédients alimentaires et 901 prescriptions de diétothérapie.

7. . On peut citer l'hôpital Tongrentang de Chengdu qui a ouvert, en 1980, une salle de restaurant proposant 96 plats médicinaux.

8. matière médicale de diététique de l'époque des Ming. Il subsiste une incertitude quant à l'identification des auteurs. On suppose que Lu He 卢和 serait à l'origine de la version initiale du texte, qui aurait été complétée ou remaniée par un ou deux autres experts. La datation de l'ouvrage n'est pas connue précisément (probablement autour de 1500). Destiné à la cour impériale, il comprend la description, en texte calligraphié, de 387 sortes d'ingrédients alimentaires et il est illustré de 492 magnifiques planches en couleurs.

9. . On traduit parfois Qi 氣 par souffle ou énergie mais ces termes ne reflètent que partiellement le sens du mot chinois qui comprend des aspects tant cosmologiques que physiologiques. Cf Eric Marié, Précis de médecine chinoise, Labège, Editions Dangles, 2008, pp.91-93.

10. . À titre d'exemples: le rhizome frais de gingembre, sudorifique, est émergent; l'igname, tonique, antidiurétique et anti-leucorrhéique est immergent ; la cardamome, anti-diarrhéique et anti-abortive, est ascendante ; la poire, laxative et antitussive, est descendante.

11. . Les fonctions de la Rate relèvent, au moins partiellement, de ce qui est attribué, en médecine occidentale, au pancréas.

12. . En médecine chinoise, le poumon est un organe unique, divisé en deux branches.

13. . Huangdi neijing 黃帝内徑 [Classique interne de l'Empereur jaune], « Yinyang yingxiang dalun 陰陽應象大論 [Grand traité sur les manifestations et correspondances du Yin/Yang] » dans Yang Weijie 楊維保 (édit.), Huangdi neijing suwen shijie 黃帝内經素問釋解 [Commentaire et explications des Questions essentielles du Classique interne de l'Empereur jaune], Taibei, Taiyan guofeng chubanshe, 1986, p. 5.

14. Selon le principe xing shi wei 形食味 [la forme se nourrit de la saveur], Ibid, p. 5.

15. Selon le principe wei shang xing 味傷形 [la saveur blesse la forme], Ibid, p. 5.

16. . On peut aussi le traduire par « méridien dont il relève ».

\section{RÉSUMÉS}

La diététique chinoise n'est pas seulement une discipline permettant d'ajuster l'alimentation aux besoins du corps, à la prévention et au traitement des maladies. Comme de nombreux aspects de la médecine chinoise, elle constitue un mode d'application des paradigmes philosophiques et cosmologiques qui prévalaient dans la Chine ancienne. Ses règles sont notamment fondées sur la nature complexe des énergies et saveurs, tropismes et actions spécifiques des ingrédients et de leurs combinaisons, ainsi que sur leurs relations avec la physiologie humaine dans la pensée médicale chinoise. Il ne s'agit pas seulement ici d'interdits ou de recommandations, de quantités ou de limites, mais d'un ballet subtil dans lequel les aliments entrent en résonnance avec climats 
et sentiments, où la nutrition participe à l'entretien des voies et demeures des souffles et à l'équilibre politique des organes qui sont autant de fonctionnaires d'un corps humain perçu à l'image de l'Empire. 